\title{
Metástase hipofisária de adenocarcinoma mamário: relato de caso
}

\section{Pituitary Metastasis of Mammary Adenocarcinoma: A Case Report}

\author{
Gabriel Chaves da Silva ${ }^{1}$ Ana Carolina Galotti ${ }^{1}$ Pedro Henrique Petit Becker ${ }^{1}$ \\ Antônio Roberto Ferreira ${ }^{1}$ Felipe Franco Pinheiro Gaia ${ }^{2}$ Antônio Fernandes Ferrari ${ }^{3}$ \\ Marco Aurélio Franco de Godoy Belfort ${ }^{4}$ \\ ${ }^{1}$ Médico Residente, Serviço de Neurocirurgia do Hospital Regional de \\ Presidente Prudente, Vila Liberdade, Pres. Prudente, SP, Brasil \\ 2 Médico Neurocirurgião, Assistente do Serviço de neurocirurgia do \\ Hospital Regional de Presidente Prudente, Vila Liberdade, Pres. \\ Prudente, SP, Brasil \\ 3 Médico Neurocirurgiao, Chefe do Serviço de Neurocirurgia do \\ Hospital Regional de Presidente Prudente, Vila Liberdade, Pres. \\ Prudente, SP, Brasil \\ ${ }^{4}$ Médico Otorrinolaringologista Assistente, Hospital Regional de \\ Presidente Prudente, Vila Liberdade, Pres. Prudente, SP, Brasil
Address for correspondence Gabriel Chaves da Silva, MR, rua Salviano Domingos Chagas, 85, apto. 13, Parque Residencial Araki, Pres. Prudente, SP, Brasil CEP: 19039-230 (e-mail: gabrielchaves2013@gmail.com). \\ Arq Bras Neurocir 2016;35:78-81.
}

\section{Resumo}

Palavras-Chave

- metástase neoplásica

- glândula hipófise

- adenocarcinoma mamário
Somente $1 \%$ de todas as cirurgias hipofisárias são realizadas para tratar tumores metastáticos desta região. Os focos primários mais comuns são respectivamente mama e pulmão. Neste artigo, descrevemos uma paciente com antecedente de adenocarcinoma mamário, que se apresenta com paralisia oculomotora súbita à direita. O objetivo do trabalho é descrever um caso de metástase mamária para a região selar, evidenciando dados clínicos e radiológicos que tornam tal diagnóstico plausível.

Only $1 \%$ of all pituitary surgeries are performed to treat metastatic tumors in this region. The most common primary sites are breast and lung respectively. In this article we describe a patient with previous breast adenocarcinoma presenting with oculomotor palsy sudden right. The objective is to describe a case of breast metastasis to the sellar region, showing clinical and radiological findings that make this plausible diagnosis.

\section{Introdução}

A região da sela túrcica pode ser acometida por uma variedade de lesões, incluindo benignas e malignas. O diagnóstico diferencial abrange adenomas hipofisários (cerca de 90\% dos casos), lesões inflamatórias, vasculares e tumores não hipofisários. As lesões metastáticas desta região são incomuns, ocorrendo em apenas $1 \%$ das ressecções da pituitária ${ }^{1}$ e 0,14 a $24 \%$ de todas as metástases cerebrais em séries de autópsia. ${ }^{2,3}$
Recebido

July 21, 2014

Aprovado

August 28, 2015

published online

January 13, 2016
DOI http://dx.doi.org/

$10.1055 / \mathrm{s}-0035-1571172$. ISSN 0103-5355.
Copyright $(\underset{2}{ } 2016$ by Thieme Publicações License terms Ltda, Rio de Janeiro, Brazil
(ब) $\Theta \circledast$ 
Provavelmente em decorrência do aumento de sobrevida e aperfeiçoamento dos exames de imagem, a frequência destas lesões tem aumentado nas últimas décadas. ${ }^{4}$ Câncer de mama e pulmão são as neoplasias metastáticas mais comuns para essa região, seguidos de próstata, células renais, trato gastrointestinal, linfomas, tireoide e plasmocitoma, muitas vezes já fazendo parte de uma disseminação generalizada, associada a outros sítios. ${ }^{5}$ Essas lesões localizam-se principalmente na hipófise posterior, provavelmente em decorrência da intensa vascularização desta região, porém podem ocorrer na hipófise anterior, sendo predominante nesta topografia a metástase mamária, devido ao tropismo hormonal destes tumors. ${ }^{8}$ Têm crescimento rápido e progressivo, provocando destruição óssea e invasão de estruturas adjacentes. ${ }^{6,7}$

Descreveremos o caso de uma mulher, branca, de 56 anos, com história de mastectomia, radioterapia e quimioterapia adjuvante em 2013, devido a adenocarcinoma invasivo de mama, que apresentou paralisia completa do terceiro nervo craniano direito, 9 meses após a cirurgia.

\section{Relato de Caso}

Paciente de 56 anos, sexo feminino, com diagnóstico de adenocarcinoma mucinoso de mama esquerda, estágio IIIB, submetida, em outro serviço, à mastectomia e linfadenectomia, recebendo posteriormente tratamento com quimioterapia, radioterapia e hormonoterapia (anastrozol). Apresentou-se, 9 meses após mastectomia, com história de ptose palpebral à direita, associada à diplopia de aparecimento súbito havia 15 dias. Ao exame neurológico, apresentava anisocoria direita, associada à paresia de reto medial direito e ptose palpebral. Negou diminuição de acuidade visual. Exames de outros aparelhos sem alterações.

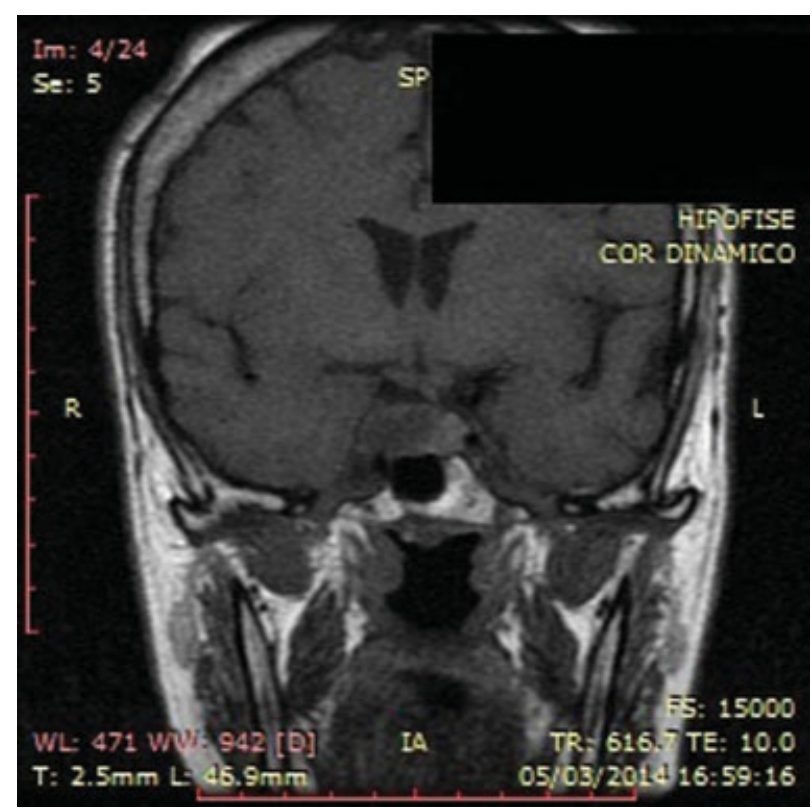

Fig. 1 RM da sequência coronal T1, evidenciando aumento nas dimensões do lobo direito da adenohipofise, com contornos lobulados e íntimo contato com o seio cavernoso direito.

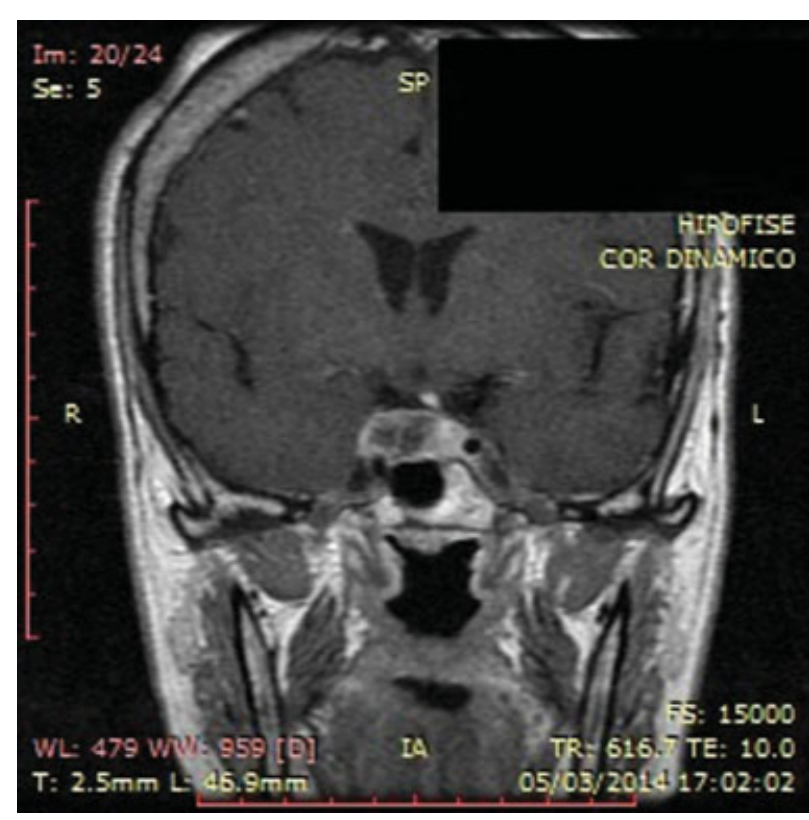

Fig. 2 RM da sequência coronal T1 pós-gadolíneo, com realce heterogêneo do lobo direito adeno-hipofisário, com invasão de estruturas intracavernosas, sem extensão suprasselar evidente.

Foi submetida inicialmente à tomografia contrastada de crânio, que evidenciou aumento volumétrico da glândula hipofisária, com destruição do arcabouço selar predominante à direita. A ressonância magnética (RM) de sela túrcica evidenciou lesão adeno-hipofisária, com contornos lobulados, de aproximadamente $1,2 \mathrm{~cm}$ no seu maior diâmetro, com aspecto ovoide, realce heterogêneo ao gadolínio, com íntimo contato com o seio cavernoso direito, sem extensão suprasselar ou compressão quiasmática (-Figs. 1, 2 and 3). A

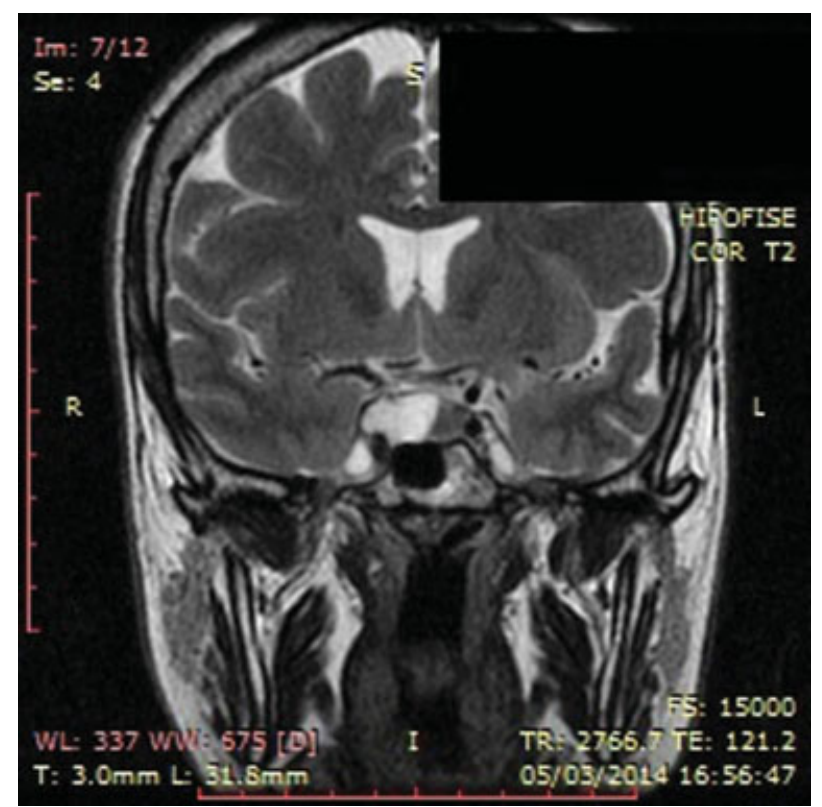

Fig. 3 RM da sequência coronal T2 evidencia nítida diferença de sinal entre a lesão metastática e hipófise normal. 
RM de crânio não evidenciou nenhuma outra lesão encefálica, e a cintilografia não mostrou disseminação óssea.

Os exames laboratoriais mostravam: prolactina, $18 \mathrm{ng} / \mathrm{dL}$ (valor de referência $[\mathrm{VR}]=3-20$ ); cortisol matutino, 20,4 (VR = 6,7-22); hormônio de crescimento, 0,31 (VR $<3,5)$; somatomedina, 144 (VR = 81-225); T4 livre, 1,20 (VR = 0,54-1,24); e hormônio tireotrópico, 0,67 ( $\mathrm{VR}=$ 0,34-5,6). Hemograma e eletrólitos sem alterações.

Optou-se, então, por acesso à lesão via transesfenoidal com técnica endoscópica. No intraoperatório, foi observada lesão de coloração castanha-clara, de consistência firme. A ressecção lesional foi subtotal, devido ao íntimo contato com o seio cavernoso direito. Não houve intercorrências durante o procedimento ou durante o pós-operatório, e a paciente recebeu alta 3 dias após a cirurgia.

Retornou ao ambulatório após 7 dias, sem novas queixas, apresentando melhora da anisocoria, porém persistência do estrabismo e da ptose palpebral.

O exame anatomopatológico e perfil imuno-histoquímico foram compatíveis com foco metastático de adenocarcinoma mucinoso de mama. A paciente foi encaminhada à oncologia, onde foi indicado tratamento radioterápico adjuvante da sela túrcica.

\section{Discussão}

Tumores metastáticos da glândula pituitária são complicações incomuns $^{9}$ (cerca de 1 a $3,6 \%$ dos tumores malignos) $^{8} \mathrm{e}$ representam 0,14 a $28,1 \%$ das metástases cerebrais. ${ }^{2,3} \mathrm{Na}$ literatura, as neoplasias mais associadas ocorrem na mama e no pulmão, correspondendo respectivamente a $30-47 \%$ e 19 $30 \%$ dos casos. ${ }^{8,9}$

A maioria das metástases se localiza na hipófise posterior (neuro-hipófise), e somente 13\%, na hipófise anterior (adeno-hipófise). Isso provavelmente ocorre devido ao aporte arterial direto da hipófise posterior (artéria hipofisária) e vascularização via sistema portal da hipófise anterior. ${ }^{10}$ Nos casos de acometimento da adeno-hipófise, deve-se suspeitar de origem mamária, provavelmente devido à afinidade hormonal desse tipo de lesão. ${ }^{8}$

A maioria das apresentações é assintomática, havendo sinais e sintomas em apenas $7 \%$ dos casos. ${ }^{2}$ Devido à topografia mais comum, é frequente a ocorrência de diabetes insípido rapidamente progressiva, associada ou não a déficits de pares cranianos, sendo os últimos geralmente associados à compressão quiasmática ou invasão do seio cavernoso. ${ }^{11}$

Não foram definidos, ainda, critérios específicos ou sensíveis que permitam a diferenciação do adenoma. As poucas características de imagem incluem: espessamento da haste hipofisária, perda do hiperssinal da hipófise posterior, isointensidade de sinal de T1 e T2, invasão do seio cavernoso e mudança esclerótica em torno da sela túrcica. ${ }^{9-12}$

Frente às dificuldades para diferenciação radiológica ou laboratorial, a história clínica do paciente com evolução de sintomas neurológicos e hormonais rapidamente progressivos, somada a algumas características radiológicas, torna-se importante para a elaboração da hipótese diagnóstica. ${ }^{12}$
Múltiplas modalidades de tratamento existem para esse tipo de lesão, incluindo ressecção cirúrgica, quimioterapia e radioterapia. ${ }^{13}$ Devido à raridade e apresentação, geralmente, em estágio final de doença metastática, não existem estudos, que comprovem a melhor forma de tratamento. ${ }^{8}$ Devido à invasão de estruturas nobres adjacentes e à alta vascularização destes tipos de tumor, a ressecção total, na maioria dos casos, é inviável. ${ }^{14}$ Atualmente, existe uma discussão quanto à radioterapia adjuvante, havendo dúvidas se a irradiação deve ser feita somente na região selar, ou em todo o crânio. ${ }^{8}$

Geralmente, quando é feito o diagnóstico de metástase nesta topografia, a doença já se encontra em estágio avançado, o que confere um prognóstico ruim para estes pacientes. Não foram encontrados estudos que comprovem a melhora da sobrevida com a abordagem cirúrgica, apesar da evidente melhora de sintomas compressivos. A sobrevida gira em torno de 6 a 22 meses, independentemente da indicação cirúrgica. ${ }^{14}$

No caso relatado, uma mulher de 56 anos, com história de câncer de mama se apresenta com paralisia súbita de oculomotor direito. A ressonância mostra lesão adeno-hipofisária e invasão do seio cavernoso ipsilateral. Frente a estes achados, a hipótese de metástase selar, apesar de infrequente, torna-se claramente plausível.

\section{Conclusão}

As metástases para a região selar são raras. Porém, devem ser consideradas como diagnóstico diferencial, principalmente em pacientes idosos, com história de malignidade ou com sintomas rapidamente progressivos de diabetes insípido e paralisia de nervos cranianos relacionados ao seio cavernoso.

Apesar do prognóstico pobre, devido à gravidade da doença sistêmica, o tratamento adequado pode aliviar os sintomas progressivos e melhorar a qualidade de vida do paciente.

\section{Referências}

1 McCormick PC, Post KD, Kandji AD, Hays AP. Metastatic carcinoma to the pituitary gland. Br J Neurosurg 1989;3(1):71-79

2 Sioutos P, Yen V, Arbit E. Pituitary gland metastases. Ann Surg Oncol 1996;3(1):94-99

3 Gsponer J, De Tribolet N, Déruaz JP, et al. Diagnosis, treatment, and outcome of pituitary tumors and other abnormal intrasellar masses. Retrospective analysis of 353 patients. Medicine (Baltimore) 1999;78(4):236-269

4 Nelson PB, Robinson AG, Martinez AJ. Metastatic tumor of the pituitary gland. Neurosurgery 1987;21(6):941-944

5 Chiang MF, Brock M, Patt S. Pituitary metastases. Neurochirurgia (Stuttg) 1990;33(4):127-131

6 Melmed S. Evaluation of pituitary masses. In: De Groot LJ, Jameson L eds. Endocrinology. 4a ed. New York: WB Saunders Company; 2001.p.282-8.

7 Albrecht S, Bilbao JM, Kovacs K. Nonpituitary tumors of the sellar region. In: Melmed S ed. The Pituitary. 2nd ed. Massachusetts: Blackwell Science Inc; 2002.p.592-608.

8 Fassett DR, Couldwell WT. Metastases to the pituitary gland. Neurosurg Focus 2004;16(4):E8

9 Komninos J, Vlassopoulou V, Protopapa D, et al. Tumors metastatic to the pituitary gland: case report and literature review. J Clin Endocrinol Metab 2004;89(2):574-580 
10 Morita A, Meyer FB, Laws ER Jr. Symptomatic pituitary metastases. J Neurosurg 1998;89(1):69-73

11 Houck WA, Olson KB, Horton J. Clinical features of tumor metastasis to the pituitary. Cancer 1970;26(3):656-659

12 Kistler M, Pribram HW. Metastatic disease of the sella turcica. Am J Roentgenol Radium Ther Nucl Med 1975;123(1):13-21
13 Losa M, Grasso M, Giugni E, Mortini P, Acerno S, Giovanelli M. Metastatic prostatic adenocarcinoma presenting as a pituitary mass: shrinkage of the lesion and clinical improvement with medical treatment. Prostate 1997;32(4):241-245

14 Freda PU, Post KD. Differential diagnosis of sellar masses. Endocrinol Metab Clin North Am 1999;28(1):81-117, vi 